\title{
LETTER
}

\section{Differences in clinical deterioration among three sub-phenotypes of COVID-19 patients at the time of first positive test: results from a clustering analysis}

\author{
Data Science Collaborative Group*
}

๑ 2020 Springer-Verlag GmbH Germany, part of Springer Nature

\section{Dear Editor,}

While studies have established risk factors for clinical deterioration in coronavirus disease 2019 (COVID-19) patients [1,2], or attempted to identify phenotypes based on experts opinion [3], identifying sub-phenotypes based on more easily obtained data could help identify patients at highest risk of clinical deterioration and refine inclusion of more homogeneous subpopulations in clinical trials. We here applied an unsupervised, multivariate clustering algorithm using easy-to-obtain clinical variables to identify COVID-19 sub-phenotypes and examined the association with clinical deterioration.

This retrospective cohort study was performed among adult COVID-19-positive patients (using real-time reverse transcriptase-polymerase chain reaction assay) with a hospital visit between February 28 and March 26, 2020, at eight teaching hospitals of the Assistance Publique-Hôpitaux de Paris. The Institutional Review Board (IRB) of Ile-de-France VII approved the study and waived the need for informed consent from individual patients (DC 2009/CO-15-000). We selected 22 candidate variables for the clustering analysis including demographic information among 608 patients with available candidate variables, disease history, major clinical symptoms, and medications on the day of positive diagnostic, which represents the final cohort (Supplementary file). We

*Correspondence: matthieu.m.legrand@gmail.com

The members of the Data Science Collaborative Group are listed in the Acknowledgements. applied an unsupervised consensus clustering method and determined the optimal number of clusters, which we also refer to as sub-phenotypes (Supplementary File) $[4,5]$. We evaluated the association between the sub-phenotypes and with clinical deterioration defined as ICU admission and/or death within 28 days. Eight hundred and ninety-three patients were enrolled (Supplementary file), 50\% required hospital admission, 104 (11.6\%) patients were treated in the ICU, and 100 (11.2\%) patients died.

We identified three distinct sub-phenotypes of patients seen at the hospitals participating in this study. Major sub-phenotype determinants are illustrated in Fig. 1 and Supplementary Fig. 2. Biological results within each subphenotype are presented in Supplementary Table 2.

Sub-phenotype \#1 $(n=179)$ included mostly younger (median age $44[\mathrm{IQR}=23.4]$ ) women $(74.9 \%)$, with no or few comorbidities (on average 0.5 comorbidity per patient) that were rarely on renin-angiotensin-aldosterone system inhibitors (RAASi) (8\%), presenting with fever (56\%), dyspnea (42\%) or cough (78\%) and numerous non-respiratory symptoms (mean 3.2/patient), including myalgia (82\%), headaches $(71 \%)$, and gastrointestinal symptoms (54\%).

Sub-phenotype \#2 $(n=279)$ included both men (54.1\%) and women, with a median age of $53[\mathrm{IQR}=26.4]$ years, with few or no comorbidities (mean 0.66/patient). Patients were rarely on RAASi (97\%). While some had respiratory symptoms (dyspnea 35\%, cough 57\%), few had non-respiratory symptoms (mean 0.8 /patient, i.e., myalgia $15 \%$, headaches $15 \%$, gastrointestinal symptoms $13 \%)$. 


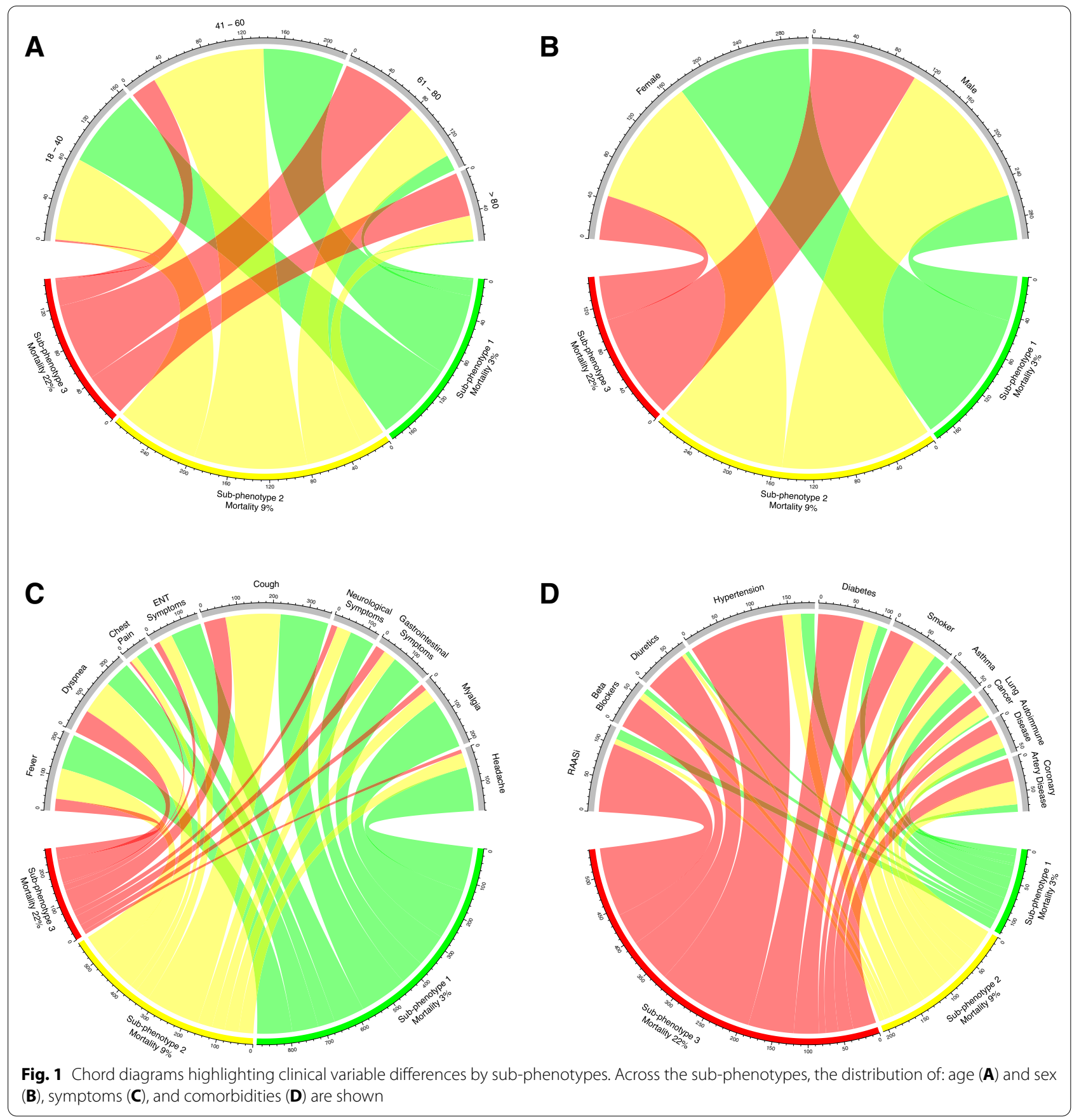

Sub-phenotype \#3 $(n=150)$ included mostly male $(70.7 \%)$ older patients (median age 73 [IQR $=19.3]$ ) with more comorbidities (mean 2.2/patient), pervasive chronic hypertension (94\%), and frequent treatment with RAASi (67\%). A minority of patients in sub-phenotype \#3 presented with fever (23\%) or pulmonary symptoms (dyspnea $45 \%$, cough $42 \%$ ), and rarely other systemic symptoms (mean 0.65/patient, i.e., myalgia $13 \%$, headaches $7 \%$, gastrointestinal symptoms $19 \%$ ).
ICU admission and/or death occurred in $8 \%, 18 \%$, and $43 \%$ of the patients in sub-phenotypes \#1, \#2, and \#3, respectively (supplementary Fig. 1). 7\%, 13\%, and 29\% of patients required ICU admission in sub-phenotype \#1, $\# 2$, and \#3, respectively. In each respective sub-phenotype, $3 \%, 9 \%$, and $22 \%$ of patients died.

In conclusion, we identified three sub-phenotypes, mostly determined by a history of chronic hypertension, the presence of fever, respiratory and non-respiratory 
symptoms, and age. These sub-phenotypes were strongly associated with clinical deterioration. The results of this clustering analysis should be now validated in other cohorts.

\section{Electronic supplementary material}

The online version of this article (https://doi.org/10.1007/s00134-020-06236-7) contains supplementary material, which is available to authorized users.

\section{Acknowledgements}

Matthieu Legrand: Department of Anesthesia and Perioperative Care, UCSF, 500 Parnassus Av, San Francisco, CA, USA; Investigation Network Initiative-Cardiovascular and Renal Clinical Trialists, French Clinical Research Infrastructure Network, Nancy, France. Rachael V. Phillips, Ivana Malenica, Lauren Eyler, Nicholas Fong: Division of Biostatistics, School of Public Health, University of California Berkeley, Berkeley, CA, USA. Alessandro Martinino, Carmen Navas Jimenez, Juan-Pablo Scarano Pereira: Hepatology service, Universite de Paris, Assistance Publique Hopitaux de Paris (AP-HP), Groupe Hospitalier Cochin Port Royal, Paris, France. Benjamin Lefevre: Service de Maladies Infectieuses, CHRU Nancy, Nancy, France. Antoine Kimmoun: Medecine Intensive et Reanimation, CHRU Nancy, Nancy, France. Bridgette LaFaye: Division of Biostatistics, School of Public Health, University of California Berkeley, Berkeley, CA, USA. Andrew Bishara: Department of Anesthesia and Perioperative Care, UCSF, 500 Parnassus Av, San Francisco, CA, USA. Alan E. Hubbard: Division of Biostatistics, School of Public Health, University of California Berkeley, Berkeley, CA, USA. Vincent Mallet: Hepatology service, Universite de Paris, Assistance Publique Hopitaux de Paris (AP-HP), Groupe Hospitalier Cochin Port Royal, Paris, France. Romain Pirracchio: Department of Anesthesia and PerioperativeCare, UCSF, 500 Parnassus Av, San Francisco, CA, USA; Investigation Network Initiative-Cardiovascular and Renal Clinical Trialists, French Clinical Research Infrastructure Network, Nancy, France.

\section{Compliance with ethical standards}

\section{Conflicts of interest}

The authors declare that they have no conflict of interest.

\section{Publisher's Note}

Springer Nature remains neutral with regard to jurisdictional claims in published maps and institutional affiliations.

Accepted: 31 August 2020

Published online: 19 October 2020

\section{References}

1. Leisman DE, Deutschman CS, Legrand M (2020) Facing COVID-19 in the ICU: vascular dysfunction, thrombosis, and dysregulated inflammation. Intensive Care Med. https://doi.org/10.1007/s00134-020-06059-6

2. Liang W, Liang H, Ou L et al (2020) Development and validation of a clinical risk score to predict the occurrence of critical illness in hospitalized patients with COVID-19. JAMA Intern Med. https://doi.org/10.1001/jamai nternmed.2020.2033

3. Clinical phenotypes of SARS-CoV-2: Implications for clinicians and researchers | European Respiratory Society. https://erj.ersjournals.com/ content/early/2020/04/20/13993003.01028-2020. Accessed 18 May 2020

4. Wilkerson MD, Hayes DN (2010) ConsensusClusterPlus: a class discovery tool with confidence assessments and item tracking. Bioinformatics 26:1572-1573. https://doi.org/10.1093/bioinformatics/btq170

5. Unbiased recursive partitioning: a conditional inference framework: J Comput Graph Stat 15(3). https://www.tandfonline.com/doi/ abs/10.1198/106186006X133933. Accessed 20 May 2020 